Pacific

Journal of

Mathematics

\title{
ON EXTENSIONS OF ALGEBRAIC GROUPS WITH FINITE QUOTIENT
}

MICHEL BRION

Volume $279 \quad$ No. 1-2

December 2015 


\title{
ON EXTENSIONS OF ALGEBRAIC GROUPS WITH FINITE QUOTIENT
}

\author{
MICHEL BRION
}

Consider an exact sequence of group schemes of finite type over a field $\boldsymbol{k}$,

$$
1 \longrightarrow N \longrightarrow G \longrightarrow Q \longrightarrow 1,
$$

where $Q$ is finite. We show that $Q$ lifts to a finite subgroup scheme $F$ of $G$; if $Q$ is étale and $k$ is perfect, then $F$ may be chosen étale as well. As applications, we obtain generalizations of classical results of Arima, Chevalley, and Rosenlicht to possibly nonconnected algebraic groups. We also show that every homogeneous space under such a group has a projective equivariant compactification.

\section{Introduction}

Consider an extension of algebraic groups, that is, an exact sequence of group schemes of finite type over a field,

$$
1 \longrightarrow N \longrightarrow G \stackrel{f}{\longrightarrow} Q \longrightarrow 1 \text {. }
$$

Such an extension is generally not split, i.e., $f$ admits no section which is a morphism of group schemes. In this note, we obtain the existence of a splitting in a weaker sense, for extensions with finite quotient group:

Theorem 1.1. Let $G$ be an algebraic group over a field $k$, and $N$ a normal subgroup of $G$ with $G / N$ finite. Then there exists a finite subgroup $F$ of $G$ such that $G=N \cdot F$.

Here $N \cdot F$ denotes, as in [SGA $3_{\text {I }}$ 1970, VIA.5.3.3], the quotient of the semidirect product $N \rtimes F$ by $N \cap F$ embedded as a normal subgroup via $x \mapsto\left(x, x^{-1}\right)$. If $G / N$ is étale and $k$ is perfect, then the subgroup $F$ may be chosen étale as well. But this fails over any imperfect field $k$, see Remark 3.3 for details.

In the case where $G$ is smooth and $k$ is perfect, Theorem 1.1 was known to Borel and Serre, and they presented a proof over an algebraically closed field of characteristic 0 (see [Borel and Serre 1964, Lemma 5.11 and footnote on p. 152]). That result was also obtained by Platonov [1966, Lemma 4.14] for smooth linear algebraic groups over perfect fields. In the latter setting, an effective version of

MSC2010: primary 14L15; secondary 14L30, $20 \mathrm{G} 15$.

Keywords: algebraic groups, finite quotients, extensions, equivariant compactifications. 
Theorem 1.1 has been obtained recently by Lucchini Arteche [2015a, Theorem 1.1]; see [Lucchini Arteche 2015b, Proposition 1.1; Chernousov et al. 2008, p. 473; Lötscher et al. 2013, Lemma 5.3] for earlier results in this direction.

Returning to an extension (1) with an arbitrary quotient $Q$, one may ask whether there exists a subgroup $H$ of $G$ such that $G=N \cdot H$ and $N \cap H$ is finite (when $Q$ is finite, the latter condition is equivalent to the finiteness of $H$ ). We then say that (1) is quasisplit, and $H$ is a quasicomplement of $N$ in $G$, with defect group $N \cap H$.

When $Q$ is smooth and $N$ is an abelian variety, every extension (1) is quasisplit (as shown by Rosenlicht [1956, Theorem 14]; see [Milne 2013, Section 2] for a modern proof). The same holds when $Q$ is reductive (i.e., $Q$ is smooth and affine, and the radical of $Q_{\bar{k}}$ is a torus), $N$ is arbitrary and $\operatorname{char}(k)=0$, as we will show in Corollary 4.8. On the other hand, the group $G$ of unipotent $3 \times 3$ matrices sits in a central extension

$$
1 \longrightarrow \mathbb{G}_{a} \longrightarrow G \longrightarrow \mathbb{G}_{a}^{2} \longrightarrow 1
$$

which is not quasisplit. It would be interesting to determine which classes of groups $N, Q$ yield quasisplit extensions. Another natural problem is to bound the defect group in terms of $N$ and $Q$. The proof of Theorem 1.1 yields some information in that direction; see Remark 3.4, and [Lucchini Arteche 2015a] for an alternative approach via nonabelian Galois cohomology.

This article is organized as follows. In Section 2, we begin the proof of Theorem 1.1 with a succession of reductions to the case where $Q=G / N$ is étale and $N$ is a smooth connected unipotent group, a torus, or an abelian variety. In Section 3, we show that every class of extensions (1) is torsion in that setting (Lemma 3.1); this quickly implies Theorem 1.1. Section 4 presents some applications of Theorem 1.1 to the structure of algebraic groups: we obtain analogues of classical results of Chevalley, Rosenlicht and Arima on smooth connected algebraic groups (see [Rosenlicht 1956; 1961; Arima 1960]) and of Mostow [1956] on linear algebraic groups in characteristic 0 . Finally, we show that every homogeneous space under an algebraic group admits a projective equivariant compactification; this result seems to have been unrecorded so far. It is well known that any such homogeneous space is quasiprojective (see [Raynaud 1970, Corollary VI.2.6]); also, the existence of equivariant compactifications of certain homogeneous spaces having no separable point at infinity has attracted recent interest (see, e.g., [Gabber 2012; Gabber et al. 2014]).

\section{Proof of Theorem 1.1: some reductions}

We first fix notation and conventions which will be used throughout this article. We consider schemes and their morphisms over a field $k$, and choose an algebraic 
closure $\bar{k}$. Given a scheme $X$ and an extension $K / k$ of fields, we denote by $X_{K}$ the $K$-scheme obtained from $X$ by the base change $\operatorname{Spec}(K) \rightarrow \operatorname{Spec}(k)$.

We use mostly [SGA $3_{\text {I }}$ 1970; SGA $3_{\text {II }}$ 1970; SGA $3_{\text {III }}$ 1970], and occasionally [Demazure and Gabriel 1970], as references for group schemes. Given such a group scheme $G$, we denote by $e_{G} \in G(k)$ the neutral element, and by $G^{0}$ the neutral component of $G$, with quotient map $\pi: G \rightarrow G / G^{0}=\pi_{0}(G)$. The group law of $G$ is denoted by $\mu: G \times G \rightarrow G,(x, y) \mapsto x y$.

Throughout this section, we consider an extension (1) and a subgroup $F$ of $G$. Then the map

$$
v: N \rtimes F \longrightarrow G, \quad(x, y) \longmapsto x y
$$

is a morphism of group schemes with kernel $N \cap F$, embedded in $N \rtimes F$ via $x \mapsto\left(x, x^{-1}\right)$. Thus, $v$ factors through a morphism of group schemes

$$
\iota: N \cdot F \longrightarrow G .
$$

Also, the composition $F \rightarrow G \rightarrow G / N$ factors through a morphism of group schemes

$$
i: F /(N \cap F) \longrightarrow G / N .
$$

By [SGA $3_{\text {I }}$ 1970, VIA.5.4], $\iota$ and $i$ are closed immersions of group schemes.

Lemma 2.1. The following conditions are equivalent:

(i) ı is an isomorphism.

(ii) $i$ is an isomorphism.

(iii) $v$ is faithfully flat.

(iv) For any scheme $S$ and $g \in G(S)$, there exists a faithfully flat morphism of finite presentation $f: S^{\prime} \rightarrow S$ and $x \in N\left(S^{\prime}\right), y \in F\left(S^{\prime}\right)$ such that $g=x y$ in $G\left(S^{\prime}\right)$.

When $G / N$ is smooth, these conditions are equivalent to:

(v) $G(\bar{k})=N(\bar{k}) F(\bar{k})$.

Proof. (i) $\Leftrightarrow$ (ii): Recall from [SGA 3 1 1970, VIA.5.5.3] that $i$ factors through an isomorphism $F /(N \cap F) \rightarrow(N \cdot F) / N$. Thus, we obtain a commutative diagram

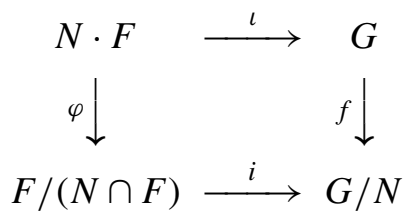

where both vertical arrows are $N$-torsors for the action of $N$ by right multiplication. As a consequence, this diagram is cartesian. In particular, $i$ is an isomorphism if and only if so is $\iota$; this yields the desired equivalence. 
(i) $\Rightarrow$ (iii): Since $v$ is identified with the quotient map of $N \rtimes F$ by $N \cap F$, the assertion follows from [SGA $3_{1}$ 1970, VIA.3.2].

(iii) $\Rightarrow$ (iv): This follows by forming the cartesian square

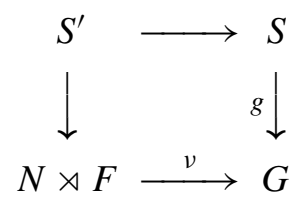

and observing that $v$ is of finite presentation, since the schemes $G, N$ and $F$ are of finite type.

(iv) $\Rightarrow$ (i): By our assumption applied to the identity map $G \rightarrow G$, there exists a scheme $S^{\prime}$ and morphisms $x: S^{\prime} \rightarrow N, y: S^{\prime} \rightarrow F$ such that the morphism $v \circ(x \times y): N \rtimes F \rightarrow G$ is faithfully flat of finite presentation. As a consequence, the morphism of structure sheaves $\mathbb{O}_{G} \rightarrow v_{*}(x \times y)_{*}\left(O_{S^{\prime}}\right)$ is injective. Thus, so are $\mathrm{O}_{G} \rightarrow v_{*}\left(\mathcal{O}_{N \rtimes F}\right)$, and hence $\mathscr{O}_{G} \rightarrow i_{*}\left(\mathcal{O}_{N \cdot F}\right)$. Since $i$ is a closed immersion, it must be an isomorphism.

(ii) $\Leftrightarrow$ (v): When $G / N$ is smooth, $i$ is an isomorphism if and only if it is surjective on $\bar{k}$-rational points. Since $(G / N)(\bar{k})=G(\bar{k}) / N(\bar{k})$ and likewise for $F /(N \cap F)$, this yields the desired equivalence.

We assume from now on that the quotient group $Q$ in the extension (1) is finite.

Lemma 2.2. If the exact sequence $1 \rightarrow H^{0} \rightarrow H \rightarrow \pi_{0}(H) \rightarrow 1$ is quasisplit for any smooth algebraic group $H$ such that $\operatorname{dim}(H)=\operatorname{dim}(G)$, then (1) is quasisplit as well.

Proof. Consider first the case where $G$ is smooth. Then $Q$ is étale, and hence $N$ contains $G^{0}$. By our assumption, there exists a finite subgroup $F \subset G$ such that $G=G^{0} \cdot F$. In view of Lemma 2.1 (iv), it follows that $G=N \cdot F$.

If $\operatorname{char}(k)=0$, then the proof is completed as every algebraic group is smooth (see, e.g., [SGA 3 1 1970, VIB.1.6.1]). So we may assume that $\operatorname{char}(k)=p>0$. Consider the $n$-fold relative Frobenius morphism

$$
F_{G}^{n}: G \longrightarrow G^{\left(p^{n}\right)}
$$

and its kernel $G_{n}$. Then $F_{G}^{n}$ is finite and bijective, so that $G_{n}$ is an infinitesimal normal subgroup of $G$. Moreover, the quotient $G / G_{n}$ is smooth for $n \gg 0$ (see [SGA $3_{\text {I }}$ 1970, VIIA.8.3]). We may thus choose $n$ so that $G / G_{n}$ and $N / N_{n}$ are smooth. The composition $N \rightarrow G \rightarrow G / G_{n}$ factors through a closed immersion of group schemes $N / N_{n} \rightarrow G / G_{n}$ by [SGA $3_{\text {I }} 1970$, VIA.5.4] again. Moreover, the image of $N / N_{n}$ is a normal subgroup of $G / G_{n}$, as follows, e.g., from our 
smoothness assumption and [SGA $3_{\text {I }}$ 1970, VIB.7.3]. This yields an exact sequence

$$
1 \longrightarrow N / N_{n} \longrightarrow G / G_{n} \longrightarrow Q^{\prime} \longrightarrow 1,
$$

where $Q^{\prime}$ is a quotient of $Q$ and hence is finite; moreover, $\operatorname{dim}\left(G / G_{n}\right)=\operatorname{dim}(G)$. By our assumption and the first step, there exists a finite subgroup $F^{\prime}$ of $G / G_{n}$ such that $G / G_{n}=\left(N / N_{n}\right) \cdot F^{\prime}$. In view of [SGA $3_{\text {I }} 1970$, VIA.5.3.1], there exists a unique subgroup $F$ of $G$ containing $G_{n}$ such that $F / G_{n}=F^{\prime}$; then $F$ is finite as well.

We check that $G=N \cdot F$ by using Lemma 2.1 (iv) again. Let $S$ be a scheme, and $g \in G(S)$. Then there exists a faithfully flat morphism of finite presentation $S^{\prime} \rightarrow S$ and $x^{\prime} \in\left(N / N_{n}\right)\left(S^{\prime}\right), y^{\prime} \in F^{\prime}\left(S^{\prime}\right)$ such that $F_{G}^{n}(g)=x^{\prime} y^{\prime}$ in $\left(G / G_{n}\right)\left(S^{\prime}\right)$. Moreover, there exists a faithfully flat morphism of finite presentation $S^{\prime \prime} \rightarrow S^{\prime}$ and $x^{\prime \prime} \in N\left(S^{\prime \prime}\right)$, $y^{\prime \prime} \in F\left(S^{\prime \prime}\right)$ such that $F_{G}^{n}\left(x^{\prime \prime}\right)=x^{\prime}$ and $F_{G}^{n}\left(y^{\prime \prime}\right)=y^{\prime}$. Then $y^{\prime \prime-1} x^{\prime \prime-1} g \in G_{n}\left(S^{\prime \prime}\right)$, and hence $g \in N\left(S^{\prime \prime}\right) F\left(S^{\prime \prime}\right)$, since $F$ contains $G_{n}$.

Remark 2.3. With the notation of the proof of Lemma 2.2, there is an exact sequence of quasicomplements

$$
1 \longrightarrow G_{n} \longrightarrow F \longrightarrow F^{\prime} \longrightarrow 1 \text {. }
$$

When $N=G^{0}$, so that $G_{n} \subset N$, we also have an exact sequence of defect groups

$$
1 \longrightarrow G_{n} \longrightarrow N \cap F \longrightarrow\left(N / G_{n}\right) \cap F^{\prime} \longrightarrow 1 \text {. }
$$

By Lemma 2.2, it suffices to prove Theorem 1.1 when $G$ is smooth and $N=G^{0}$, so that $Q=\pi_{0}(G)$. We may thus choose a maximal torus $T$ of $G$ (see [SGA $3_{\text {II }} 1970$, XIV.1.1]). Then the normalizer $N_{G}(T)$ and the centralizer $Z_{G}(T)$ are (represented by) subgroups of $G$ (see [SGA $3_{\text {I }} 1970$, VIB.6.2.5]). Moreover, $N_{G}(T)$ is smooth by [SGA $3_{\text {II }} 1970$, XI.2.4]. We now gather further properties of $N_{G}(T)$ :

Lemma 2.4. (i) $G=G^{0} \cdot N_{G}(T)$.

(ii) $N_{G}(T)^{0}=Z_{G^{0}}(T)$.

(iii) We have an exact sequence $1 \rightarrow W\left(G^{0}, T\right) \rightarrow \pi_{0}\left(N_{G}(T)\right) \rightarrow \pi_{0}(G) \rightarrow 1$, where $W\left(G^{0}, T\right):=N_{G^{0}}(T) / Z_{G^{0}}(T)=\pi_{0}\left(N_{G^{0}}(T)\right)$ denotes the Weyl group.

Proof. (i) By Lemma 2.1 (v), it suffices to show that $G(\bar{k})=G^{0}(\bar{k}) N_{G}(T)(\bar{k})$. Let $x \in G(\bar{k})$, then $x T x^{-1}$ is a maximal torus of $G^{0}(\bar{k})$, and hence $x T x^{-1}=y T y^{-1}$ for some $y \in G^{0}(\bar{k})$. Thus, $x \in y N_{G}(T)(\bar{k})$, which yields the assertion.

(ii) We may assume that $k$ is algebraically closed and $G$ is connected (since $\left.N_{G}(T)^{0}=N_{G^{0}}(T)^{0}\right)$. Then $Z_{G}(T)$ is a Cartan subgroup of $G$, and hence equals its connected normalizer by [SGA $3_{\text {II }}$ 1970, XII.6.6].

(iii) By (i), the natural map $N_{G}(T) / N_{G^{0}}(T) \rightarrow \pi_{0}(G)$ is an isomorphism. Combined with (ii), this yields the statement. 
Remark 2.5. If $N_{G}(T)=N_{G}(T)^{0} \cdot F$ for some subgroup $F \subset N_{G}(T)$, then by Lemmas 2.1 and 2.4, $G=G^{0} \cdot F$. Moreover, the commutative diagram of exact sequences

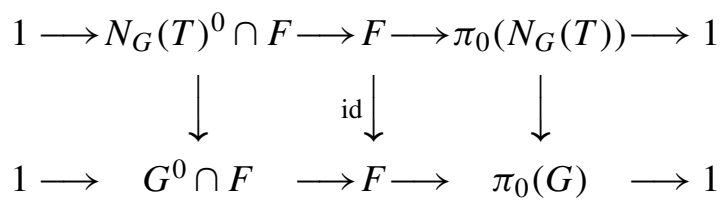

together with Lemma 2.4 yields the exact sequence

$$
1 \longrightarrow Z_{G^{0}}(T) \cap F \longrightarrow G^{0} \cap F \longrightarrow W\left(G^{0}, T\right) \longrightarrow 1 .
$$

In view of Lemma 2.1 (iv) and Lemma 2.4 (i), it suffices to prove Theorem 1.1 under the additional assumption that $T$ is normal in $G$. Then $T$ is central in $G^{0}$, and hence $G_{\bar{k}}^{0}$ is nilpotent by [SGA $3_{\text {II }} 1970$, XII.6.7]. It follows that $G^{0}$ is nilpotent, in view of [SGA 3 I 1970, VIB.8.3]. To obtain further reductions, we will use the following:

Lemma 2.6. Let $N^{\prime}$ be a normal subgroup of $G$ contained in $N$. Assume that the resulting exact sequence $1 \rightarrow N / N^{\prime} \rightarrow G / N^{\prime} \rightarrow Q \rightarrow 1$ is quasisplit, and that any exact sequence of algebraic groups $1 \rightarrow N^{\prime} \rightarrow G^{\prime} \rightarrow Q^{\prime} \rightarrow 1$, where $Q^{\prime}$ is finite, is quasisplit as well. Then (1) is quasisplit.

Proof. By assumption, there exists a finite subgroup $F^{\prime}$ of $G / N^{\prime}$ for which $G / N^{\prime}=\left(N / N^{\prime}\right) \cdot F^{\prime}$. Denote by $G^{\prime}$ the subgroup of $G$ containing $N^{\prime}$ such that $G^{\prime} / N^{\prime}=F^{\prime}$. By assumption again, there is a finite subgroup $F$ of $G^{\prime}$ containing $N^{\prime}$ such that $G^{\prime}=N^{\prime} \cdot F$. We check that $G=N \cdot F$ using Lemma 2.1 (iv). Let $S$ be a scheme, and $g \in G(S)$; denote by $f^{\prime}: G \rightarrow G / N^{\prime}$ the quotient map. Then there exists a faithfully flat morphism of finite presentation $S^{\prime} \rightarrow S$ and $x \in\left(N / N^{\prime}\right)\left(S^{\prime}\right)$, $y \in F^{\prime}\left(S^{\prime}\right)$ such that $f^{\prime}(g)=x y$ in $\left(G / N^{\prime}\right)\left(S^{\prime}\right)$. Moreover, there exists a faithfully flat morphism of finite presentation $S^{\prime \prime} \rightarrow S^{\prime}$ and $z \in N\left(S^{\prime \prime}\right), w \in G^{\prime}\left(S^{\prime \prime}\right)$ such that $f^{\prime}(z)=x$ and $f^{\prime}(w)=y$. Then $w^{-1} z^{-1} g \in N^{\prime}\left(S^{\prime \prime}\right)$, and hence $g \in N\left(S^{\prime \prime}\right) G^{\prime}\left(S^{\prime \prime}\right)$, as $G^{\prime}$ contains $N^{\prime}$. This shows that $G=N \cdot G^{\prime}=N \cdot\left(N^{\prime} \cdot F\right)$. We conclude by observing that $N \cdot\left(N^{\prime} \cdot F\right)=N \cdot F$, in view of Lemma 2.1 (iv) again.

Remark 2.7. With the notation of the proof of Lemma 2.6, we have an exact sequence $1 \rightarrow N^{\prime} \rightarrow G^{\prime}=N^{\prime} \cdot F \rightarrow F^{\prime} \rightarrow 1$, and hence an exact sequence of quasicomplements

$$
1 \longrightarrow N^{\prime} \cap F \longrightarrow F \longrightarrow F^{\prime} \longrightarrow 1 \text {. }
$$

Moreover, we obtain an exact sequence $1 \rightarrow N^{\prime} \rightarrow N \cap G^{\prime} \rightarrow\left(N / N^{\prime}\right) \cap F^{\prime} \rightarrow 1$, by using [SGA 3 1 1970, VIA.5.3.1]. Since $N \cap G^{\prime}=N \cap\left(N^{\prime} \cdot F\right)=N^{\prime} \cdot(N \cap F)$, where the latter equality follows from Lemma 2.1 (iv), this yields an exact sequence 
of defect groups

$$
1 \longrightarrow N^{\prime} \cap F \longrightarrow N \cap F \longrightarrow\left(N / N^{\prime}\right) \cap F^{\prime} \longrightarrow 1 .
$$

Next, we show that it suffices to prove Theorem 1.1 when $G^{0}$ is assumed in addition to be commutative.

We argue by induction on the dimension of $G$ (assumed to be smooth, with $G^{0}$ nilpotent). If $\operatorname{dim}(G)=1$, then $G^{0}$ is either a $k$-form of $\mathbb{G}_{a}$ or $\mathbb{G}_{m}$, or an elliptic curve; in particular, $G^{0}$ is commutative. In higher dimensions, the derived subgroup $D\left(G^{0}\right)$ is a smooth, connected normal subgroup of $G$ contained in $G^{0}$, and the quotient $G^{0} / D\left(G^{0}\right)$ is commutative of positive dimension (see [SGA 3 I 1970, VIB.7.8, 8.3]). Moreover, $G / D\left(G^{0}\right)$ is smooth, and $\pi_{0}\left(G / D\left(G^{0}\right)\right)=\pi_{0}(G)$. By the induction assumption, it follows that the exact sequence

$$
1 \longrightarrow G^{0} / D\left(G^{0}\right) \longrightarrow G / D\left(G^{0}\right) \longrightarrow \pi_{0}(G) \longrightarrow 1
$$

is quasisplit. Also, every exact sequence $1 \rightarrow D\left(G^{0}\right) \rightarrow G^{\prime} \rightarrow Q^{\prime} \rightarrow 1$, where $Q^{\prime}$ is finite, is quasisplit, by the induction assumption again together with Lemma 2.2. Thus, Lemma 2.6 yields the desired reduction.

We now show that we may further assume $G^{0}$ to be a torus, a smooth connected commutative unipotent group, or an abelian variety.

Indeed, we have an exact sequence of commutative algebraic groups

$$
1 \longrightarrow T \longrightarrow G^{0} \longrightarrow H \longrightarrow 1,
$$

where $T$ is the maximal torus of $G^{0}$, and $H$ is smooth and connected. Moreover, we have an exact sequence

$$
1 \longrightarrow H_{1} \longrightarrow H \longrightarrow H_{2} \longrightarrow 1,
$$

where $H_{1}$ is a smooth connected affine algebraic group, and $H_{2}$ is a pseudoabelian variety in the sense of [Totaro 2013], i.e., $\mathrm{H}_{2}$ has no nontrivial smooth connected affine normal subgroup. Since $H_{1}$ contains no nontrivial torus, it is unipotent; also, $H_{2}$ is an extension of a smooth connected unipotent group by an abelian variety $A$, in view of [Totaro 2013, Theorem 2.1]. Note that $T$ is a normal subgroup of $G$ (the largest subtorus). Also, $H_{1}$ is a normal subgroup of $G / T$ (the largest smooth connected affine normal subgroup of the neutral component), and $A$ is a normal subgroup of $(G / T) / H_{1}$ as well (the largest abelian subvariety). Thus, arguing by induction on the dimension as in the preceding step, with $D\left(G^{0}\right)$ replaced successively by $T, H_{1}$ and $A$, yields our reduction.

When $G^{0}$ is unipotent and $\operatorname{char}(k)=p>0$, we may further assume that $G^{0}$ is killed by $p$. Indeed, by [SGA $3_{\text {II }}$ 1970, XVII.3.9], there exists a composition series $\left\{e_{G}\right\}=G_{0} \subset G_{1} \subset \cdots \subset G_{n}=G^{0}$ such that each $G_{i}$ is normal in $G$, and each 
quotient $G_{i} / G_{i-1}$ is a $k$-form of some $\left(\mathbb{G}_{a}\right)^{r_{i}}$; in particular, $G_{i} / G_{i-1}$ is killed by $p$. Our final reduction follows by induction on $n$.

\section{Proof of Theorem 1.1: extensions by commutative groups}

In this section, we consider smooth algebraic groups $Q, N$ such that $Q$ is finite and $N$ is commutative. Given an extension (1), the action of $G$ on $N$ by conjugation factors through an action of $Q$ by group automorphisms, which we denote by $(x, y) \mapsto y^{x}$, where $x \in Q$ and $y \in N$. Recall that the isomorphism classes of such extensions with a prescribed $Q$-action on $N$ form a commutative (abstract) group, which we denote by $\operatorname{Ext}^{1}(Q, N)$; see [SGA $3_{\text {II }}$ 1970, XVII.App. I] (and [Demazure and Gabriel 1970, III.6.1] for the setting of extensions of group sheaves).

Lemma 3.1. With the above notation and assumptions, the group $\operatorname{Ext}^{1}(Q, N)$ is torsion.

Proof. Any extension (1) yields an $N$-torsor over $Q$ for the étale topology, since $Q$ is finite and étale. This defines a map $\tau: \operatorname{Ext}^{1}(Q, N) \rightarrow H_{\text {et }}^{1}(Q, N)$, which is a group homomorphism (indeed, the sum of any two extensions is obtained by taking their direct product, pulling back under the diagonal map $Q \rightarrow Q \times Q$, and pushing forward under the multiplication $N \times N \rightarrow N$; and the sum of any two torsors is obtained by the analogous operations). The kernel of $\tau$ consists of those classes of extensions that admit a section (which is a morphism of schemes). In view of [SGA $3_{\text {II }}$ 1970, XVII.App. I.3.1], this yields an exact sequence

$$
0 \longrightarrow H^{2}(Q, N) \longrightarrow \operatorname{Ext}^{1}(Q, N) \stackrel{\tau}{\longrightarrow} H_{\text {ét }}^{1}(Q, N),
$$

where $H H^{i}$ stands for Hochschild cohomology (denoted by $H^{i}$ in [SGA $3_{\text {I }}$ 1970; SGA $3_{\text {II }}$ 1970; SGA $3_{\text {III }}$ 1970], and by $H_{0}^{i}$ in [Demazure and Gabriel 1970]). Moreover, the group $H_{\text {ét }}^{1}(Q, N)$ is torsion (as follows, e.g., from [Rosenlicht 1956, Theorem 14]), and $H H^{2}(Q, N)$ is killed by the order of $Q$, as a special case of [SGA $3_{\text {II }}$ 1970, XVII.5.2.4].

Remark 3.2. The above argument yields that $\operatorname{Ext}^{1}(Q, N)$ is killed by $m d$ if $Q$ is finite étale of order $m$, and $N$ is a torus split by an extension of $k$ of degree $d$. Indeed, we just saw that $H H^{2}(Q, N)$ is killed by $m$; also, $H_{\text {ét }}^{1}(Q, N)$ is a direct sum of groups of the form $H_{\text {ét }}^{1}\left(\operatorname{Spec}\left(k^{\prime}\right), N\right)$ for finite separable extensions $k^{\prime}$ of $k$, and these groups are killed by $d$. This yields a slight generalization of [Lucchini Arteche 2015b, Proposition 1.1], via a different approach.

End of the proof of Theorem 1.1. Recall from our reductions in Section 2 that we may assume $G^{0}$ to be a smooth commutative unipotent group, a torus, or an abelian variety. We will rather denote $G^{0}$ by $N$, and $\pi_{0}(G)$ by $Q$. 
We first assume in addition that $\operatorname{char}(k)=0$ if $N$ is unipotent. Then the $n$-th power map

$$
n_{N}: N \longrightarrow N, \quad x \longmapsto x^{n}
$$

is an isogeny for any positive integer $n$. Consider an extension (1) and denote by $\gamma$ its class in $\operatorname{Ext}^{1}(Q, N)$. By Lemma 3.1, we may choose $n$ so that $n \gamma=0$. Also, $n \gamma=\left(n_{N}\right)_{*}(\gamma)$ (the pushout of $\gamma$ by $\left.n_{N}\right)$; moreover, the exact sequence of commutative algebraic groups

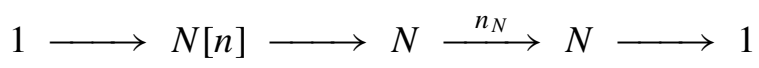

yields an exact sequence

$$
\operatorname{Ext}^{1}(Q, N[n]) \longrightarrow \operatorname{Ext}^{1}(Q, N) \stackrel{\left(n_{N}\right)_{*}}{\longrightarrow} \operatorname{Ext}^{1}(Q, N)
$$

due to [SGA $3_{\text {II }}$ 1970, XVII.App. I.2.1]. Thus, there is a class $\gamma^{\prime} \in \operatorname{Ext}^{1}(Q, N[n])$ with pushout $\gamma$, i.e., we have a commutative diagram of extensions

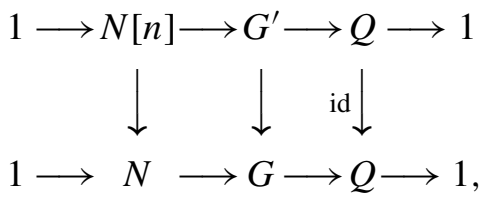

where the square on the left is cartesian. It follows that $G^{\prime}$ is a finite subgroup of $G$, and $G=N \cdot G^{\prime}$.

Next, we consider the remaining case, where $N$ is unipotent and $\operatorname{char}(k)=p>0$. In view of our final reduction at the end of Section 2, we may further assume that $N$ is killed by $p$. Then there exists an étale isogeny $N \rightarrow N_{1}$, where $N_{1}$ is a vector group (see [Conrad et al. 2015, Lemma B.1.10]). This yields another commutative diagram of extensions

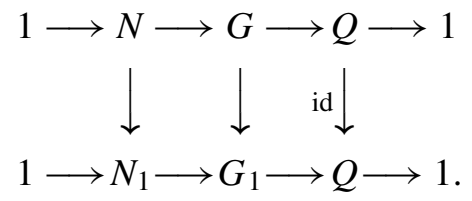

Assume that there exists a finite subgroup $F_{1}$ of $G_{1}$ such that $G_{1}=N_{1} \cdot F_{1}$. Let $F$ be the pullback of $F_{1}$ to $G$; then $F$ is a finite subgroup, and one checks that $G=N \cdot F$ using Lemma 2.1 (iv). Thus, we may finally assume that $N$ is a vector group.

Under that assumption, the $N$-torsor $G \rightarrow Q$ is trivial, since $Q$ is affine. Thus, we may choose a section $s: Q \rightarrow G$. Also, we may choose a finite Galois extension of fields $K / k$ such that $Q_{K}$ is constant. Then $s$ yields a section $s_{K}: Q_{K} \rightarrow G_{K}$, equivariant under the Galois group $\Gamma_{K}:=\mathrm{Gal}(K / k)$. So we may view $G(K)$ as the set of the $y s(x)$, where $y \in N(K)$ and $x \in Q(K)$, with multiplication

$$
y s(x) y^{\prime} s\left(x^{\prime}\right)=y y^{\prime x} c\left(x, x^{\prime}\right) s\left(x x^{\prime}\right),
$$


where $c \in Z^{2}(Q(K), N(K))^{\Gamma_{K}}$. Consider the (abstract) subgroup $H \subset N(K)$ generated by the $c\left(x^{\prime}, x^{\prime \prime}\right)^{x}$, where $x, x^{\prime}, x^{\prime \prime} \in Q(K)$. Then $H$ is finite, since $N(K)$ is killed by $p$ and $Q(K)$ is finite. Moreover, $H s(Q(K))$ is a subgroup of $G(K)$, in view of the above formula for the multiplication. Clearly, $H s(Q(K))$ is finite and stable under $\Gamma_{K}$; thus, it corresponds to a finite (algebraic) subgroup $G^{\prime}$ of $G$. Also, we obtain as above that $G=N \cdot G^{\prime}$. This completes the proof of Theorem 1.1.

Remark 3.3. If $k$ is perfect, then the subgroup $F$ as in Theorem 1.1 may be chosen étale. Indeed, the reduced subscheme $F_{\text {red }}$ is then a subgroup by [SGA $3_{\text {I }} 1970$, VIA.0.2]. Moreover, $G(\bar{k})=G^{0}(\bar{k}) F_{\text {red }}(\bar{k})$, and hence $G=G^{0} \cdot F_{\text {red }}$ in view of Lemma $2.1(\mathrm{v})$.

In contrast, when $k$ is imperfect, there exists a finite group $G$ admitting no étale subgroup $F$ such that $G=G^{0} \cdot F$. Consider for example (as in [SGA 3 I 1970, VIA.1.3.2]) the subgroup $G$ of $\mathbb{G}_{a, k}$ defined by the additive polynomial $X^{p^{2}}-t X^{p}$, where $p:=\operatorname{char}(k)$ and $t \in k \backslash k^{p}$. Then $G$ has order $p^{2}$ and $G^{0}$ has order $p$. If $G=G^{0} \cdot F$ with $F$ étale, then $G^{0} \cap F$ is trivial. Thus, $G \cong G^{0} \rtimes F$ and $F$ has order $p$. Let $K:=k\left(t^{1 / p}\right)$. Then $F_{K}$ is contained in $\left(G_{K}\right)_{\text {red }}$, which is the subgroup of $\mathbb{G}_{a, K}$ defined by the additive polynomial $X^{p}-t^{1 / p} X$. By counting dimensions, it follows that $F_{K}=\left(G_{K}\right)_{\text {red }}$, which yields a contradiction as $\left(G_{K}\right)_{\text {red }}$ is not defined over $k$.

Remark 3.4. One may obtain information on the defect group $N \cap F$ by examining the steps in the proof of Theorem 1.1 and combining Remarks 2.3, 2.5 and 2.7. For instance, if $G$ is smooth, then $N \cap F$ is an extension of the Weyl group $W\left(G^{0}, T\right)$ by the nilpotent group $Z_{G^{0}}(T) \cap F$, where $T$ is a maximal torus of $G$. If $\operatorname{char}(k)=0$ (so that $G$ is smooth), then $Z_{G^{0}}(T) \cap F$ is commutative. Indeed, $Z_{G^{0}}(T)$ is a connected nilpotent algebraic group, and hence an extension of a semiabelian variety $S$ by a connected unipotent algebraic group $U$. Thus, $U \cap F$ is trivial, and hence $Z_{G^{0}}(T) \cap F$ is isomorphic to a subgroup of $S$.

Remark 3.5. When $k$ is finite, Theorem 1.1 follows readily from our first reduction step (Lemma 2.2) together with a theorem of Lang [1956, Theorem 2]. More specifically, let $H$ be a smooth algebraic group and choose representatives $x_{1}, \ldots, x_{m}$ of the orbits of the Galois group $\Gamma:=\operatorname{Gal}(\bar{k} / k)$ in $\pi_{0}(H)(\bar{k})$. Denote by $\Gamma_{i} \subset \Gamma$ the isotropy group of $x_{i}$ and set $k_{i}:=\bar{k}^{\Gamma_{i}}$ for $i=1, \ldots, m$. Then $x_{i} \in \pi_{0}(H)\left(k_{i}\right)$, and hence the fiber $\pi_{k_{i}}^{-1}\left(x_{i}\right)$ (a torsor under $H_{k_{i}}^{0}$ ) contains a $k_{i}$-rational point. Consider the subfield

$$
K:=\prod_{i=1}^{n} k_{i} \subset \bar{k} .
$$

Then the finite étale group scheme $\pi_{0}(H)_{K}$ is constant, and $\pi$ is surjective on $K$-rational points. Thus, $\pi_{0}(H)$ has a quasicomplement in $H$ : the finite étale group scheme corresponding to the constant, $\Gamma$-stable subgroup scheme $H(K)$ of $H_{K}$. 


\section{Some applications}

We first recall two classical results on the structure of algebraic groups. The first one is the affinization theorem (see [Demazure and Gabriel 1970, III.3.8] and also [SGA 3 I 1970, VIB.12.2]): any algebraic group $G$ has a smallest normal subgroup $H$ such that $G / H$ is affine. Moreover, $H$ is smooth, connected and contained in the center of $G^{0}$; we have $\mathcal{O}(H)=k$ (such an algebraic group is called antiaffine) and $\mathrm{O}(G / H)=\mathcal{O}(G)$.

Consequently, $H$ is the fiber at $e_{G}$ of the affinization morphism $G \rightarrow \operatorname{Spec} O(G)$; moreover, the formation of $H$ commutes with arbitrary field extensions. Also, note that $H$ is the largest antiaffine subgroup of $G$; we will denote $H$ by $G_{\text {ant }}$. The structure of antiaffine groups is described in [Brion 2009] and [Sancho and Sancho 2009].

The second structure result is a version of a theorem of Chevalley, due to Raynaud [1970, Lemma IX.2.7] (see also [Bosch et al. 1990, 9.2 Theorem 1]): any connected algebraic group $G$ has a smallest affine normal subgroup $N$ such that $G / N$ is an abelian variety. Moreover, $N$ is connected; if $G$ is smooth and $k$ is perfect, then $N$ is smooth as well. We will denote $N$ by $G_{\text {aff }}$.

We will also need the following observation:

Lemma 4.1. Let $G$ be an algebraic group, and $N$ a normal subgroup. Then the quotient map $f: G \rightarrow G / N$ yields an isomorphism $G_{\text {ant }} /\left(G_{\text {ant }} \cap N\right) \cong(G / N)_{\text {ant }}$.

Proof. We have a closed immersion of group schemes $G_{\text {ant }} /\left(G_{\text {ant }} \cap N\right) \rightarrow G / N$; moreover, $G_{\text {ant }} /\left(G_{\text {ant }} \cap N\right)$ is antiaffine. So we obtain a closed immersion of commutative group schemes $i: G_{\text {ant }} /\left(G_{\text {ant }} \cap N\right) \rightarrow(G / N)$ ant. The cokernel of $i$ is antiaffine, as a quotient of $(G / N)_{\text {ant }}$. Also, this cokernel is a subgroup of $(G / N) /\left(G_{\text {ant }} /\left(G_{\text {ant }} \cap N\right)\right)$, which is a quotient of $G / G_{\text {ant }}$. Since the latter is affine, it follows that $\operatorname{Coker}(i)$ is affine as well, by using [SGA 3 I 1970, VIB.11.17]. Thus, $\operatorname{Coker}(i)$ is trivial, i.e., $i$ is an isomorphism.

We now obtain a further version of Chevalley's structure theorem, for possibly nonconnected algebraic groups:

Theorem 4.2. Any algebraic group $G$ has a smallest affine normal subgroup $N$ such that $G / N$ is proper. Moreover, $N$ is connected.

Proof. It suffices to show that $G$ admits an affine normal subgroup $N$ such that $G / N$ is proper. Indeed, given another such subgroup $N^{\prime}$, the natural map

$$
G /\left(N \cap N^{\prime}\right) \longrightarrow G / N \times G / N^{\prime}
$$

is a closed immersion, and hence $G /\left(N \cap N^{\prime}\right)$ is proper. Taking for $N$ a minimal such subgroup, it follows that $N$ is the smallest one. Moreover, the natural morphism $G / N^{0} \rightarrow G / N$ is finite, since it is a torsor under the finite group $N / N^{0}$ (see 
[SGA $3_{\text {I }}$ 1970, VIA.5.3.2]). As a consequence, $G / N^{0}$ is proper; hence $N=N^{0}$ by the minimality assumption. Thus, $N$ is connected.

Also, we may reduce to the case where $G$ is smooth by using the relative Frobenius morphism as in the proof of Lemma 2.2.

If in addition $G$ is connected, then we just take $N=G_{\text {aff. In the general case, we }}$ consider the (possibly nonnormal) subgroup $H:=\left(G^{0}\right)_{\text {aff }}$; then the homogeneous space $G / H$ is proper, since $G / G^{0}$ is finite and $G^{0} / H$ is proper. As a consequence, the automorphism functor of $G / H$ is represented by a group scheme Aut $_{G / H}$, locally of finite type; in particular, the neutral component $\mathrm{Aut}_{G / H}^{0}$ is an algebraic group (see [Matsumura and Oort 1967, Theorem 3.7]). The action of $G$ by left multiplication on $G / H$ yields a morphism of group schemes

$$
\varphi: G \longrightarrow \operatorname{Aut}_{G / H}
$$

The kernel $N$ of $\varphi$ is a closed subscheme of $H$, and hence is affine. To complete the proof, it suffices to show that $G / N$ is proper. In turn, it suffices to check that $(G / N)^{0}$ is proper. Since $(G / N)^{0} \cong G^{0} /\left(G^{0} \cap N\right)$, and $G^{0} \cap N$ is the kernel of the restriction $G^{0} \rightarrow \operatorname{Aut}_{G / H}^{0}$, we are reduced to showing that Aut $_{G / H}^{0}$ is proper (by using [SGA $3_{\text {I }}$ 1970, VIA.5.4.1] again).

We claim that Aut $_{G / H}^{0}$ is an abelian variety. Indeed, $(G / H)_{\bar{k}}$ is a finite disjoint union of copies of $\left(G^{0} / H\right)_{\bar{k}}$, which is an abelian variety. Also, the natural morphism $A \rightarrow \mathrm{Aut}_{A}^{0}$ is an isomorphism for any abelian variety $A$. Thus, $\left(\operatorname{Aut}_{G / H}^{0}\right)_{\bar{k}}$ is an abelian variety (a product of copies of $\left.\left(G^{0} / H\right)_{\bar{k}}\right)$; this yields our claim, and completes the proof.

Remark 4.3. The formation of $G_{\text {aff }}$ (for a connected group scheme $G$ ) commutes with separable algebraic field extensions, as follows from a standard argument of Galois descent. But this formation does not commute with purely inseparable field extensions, in view of [SGA $3_{\text {II }}$ 1970, XVII.C.5].

Likewise, the formation of $N$ as in Theorem 4.2 commutes with separable algebraic field extensions. As a consequence, $N=\left(G^{0}\right)$ aff for any smooth group scheme $G$ (since $\left(G^{0}\right)$ aff is invariant under any automorphism of $G$, and hence is a normal subgroup of $G$ when $k$ is separably closed). In particular, if $k$ is perfect and $G$ is smooth, then $N$ is smooth as well.

For an arbitrary group scheme $G$, we may have $N \neq\left(G^{0}\right)_{\text {aff }}$, e.g., when $G$ is infinitesimal: then $N$ is trivial, while $\left(G^{0}\right)_{\text {aff }}=G$.

We do not know if the formations of $G_{\text {aff }}$ and $N$ commute with arbitrary separable field extensions.

The structure of proper algebraic groups is easily described as follows:

Proposition 4.4. Given a proper algebraic group $G$, there exists an abelian variety $A$, a finite group $F$ equipped with an action $F \rightarrow$ Aut $_{A}$ and a normal subgroup 
$D \subset F$ such that $D$ acts faithfully on $A$ by translations and $G \cong(A \rtimes F) / D$, where $D$ is embedded in $A \rtimes F$ via $x \mapsto\left(x, x^{-1}\right)$. Moreover, $A=G_{\text {ant }}$ and $F / D \cong G / G_{\text {ant }}$ are uniquely determined by $G$. Finally, $G$ is smooth if and only if $F / D$ is étale.

Proof. Note that $G_{\text {ant }}$ is a smooth connected proper algebraic group, and hence an abelian variety. Moreover, the quotient group $G / G_{\text {ant }}$ is affine and proper, hence finite. By Theorem 1.1, there exists a finite subgroup $F \subset G$ such that $G=G_{\text {ant }} \cdot F$. In particular, $G \cong\left(F \ltimes G_{\text {ant }}\right) /\left(F \cap G_{\text {ant }}\right)$; this implies the existence assertion. For the uniqueness, just note that $\mathcal{O}(G) \cong \mathbb{O}(G / A) \cong \mathscr{O}(F / D)$, and this identifies the affinization morphism to the natural homomorphism $G \rightarrow F / D$, with kernel $A$.

If $G$ is smooth, then so is $G / A \cong F / D$; as $F / D$ is finite, it must be étale. Since the homomorphism $G \rightarrow F / D$ is smooth, the converse holds as well.

Remark 4.5. The simplest examples of proper algebraic groups are the semidirect products $G=A \rtimes F$, where $F$ is a finite group acting on the abelian variety $A$. If this action is nontrivial (for example, if $A$ is nontrivial and $F$ is the constant group $\mathbb{Z} / 2 \mathbb{Z}$ acting via $x \mapsto x^{ \pm 1}$ ), then every morphism of algebraic groups $f: G \rightarrow H$, where $H$ is connected, has a nontrivial kernel. (Otherwise, $A$ is contained in the center of $G$ by the affinization theorem.) This yields examples of algebraic groups which admit no faithful representation in a connected algebraic group.

Remark 4.6. With the notation and assumptions of Proposition 4.4, consider a subgroup $H \subset G$ and the homogeneous space $X:=G / H$. Then there exists an abelian variety $B$ quotient of $A$, a subgroup $I \subset F$ containing $D$, and a faithful homomorphism $I \rightarrow \operatorname{Aut}_{B}$ such that the scheme $X$ is isomorphic to the associated fiber bundle $F \times{ }^{I} B$. Moreover, the schemes $F / I$ and $B$ are uniquely determined by $X$, and $X$ is smooth if and only if $F / I$ is étale.

Indeed, let $K:=A \cdot H$, then $X \cong G \times{ }^{K} K / H \cong F \times{ }^{I} K / H$, where $I:=F \cap K$. Moreover, $K / H \cong A /(A \cap H)$ is an abelian variety. This shows the existence assertion; those on uniqueness and smoothness are checked as in the proof of Proposition 4.4.

Conversely, given a finite group $F$ and a subgroup $I \subset F$ acting on an abelian variety $B$, the associated fiber bundle $F \times{ }^{I} B$ exists (since it is the quotient of the projective scheme $F \times B$ by the finite group $I$ ), and is homogeneous whenever $F / I$ is étale (since $\left(F \times{ }^{I} B\right)_{\bar{k}}$ is just a disjoint union of copies of $\left.B_{\bar{k}}\right)$. We do not know how to characterize the homogeneity of $F \times{ }^{I} B$ when the quotient $F / I$ is arbitrary.

Returning to an arbitrary algebraic group $G$, we have the "Rosenlicht decomposition" $G=G_{\text {ant }} \cdot G_{\text {aff }}$ when $G$ is smooth and connected (see, e.g., [Brion 2009]). We now extend this result to possibly nonconnected groups:

Theorem 4.7. Let $G$ be an algebraic group. Then there exists an affine subgroup $H$ of $G$ such that $G=G_{\text {ant }} \cdot H$. If $G$ is smooth and $k$ is perfect, then $H$ may be chosen smooth. 
Proof. By Theorem 4.2, we may choose an affine normal subgroup $N \subset G$ such that $G / N$ is proper. In view of Proposition 4.4, there exists a finite subgroup $F$ of $G / N$ such that $G / N=(G / N)_{\text {ant }} \cdot F$, and $(G / N)_{\text {ant }}$ is an abelian variety. Let $H$ be the subgroup of $G$ containing $N$ such that $G / H=F$. Then $H$ is affine, since it sits in an extension $1 \rightarrow N \rightarrow H \rightarrow F \rightarrow 1$. We check that $G=G_{\text {ant }} \cdot H$ by using Lemma 2.1 (iv). Let $S$ be a scheme, and $g \in G(S)$. Denote by $g^{\prime}$ the image of $g$ in $(G / N)(S)$. Then there exist a faithfully flat morphism of finite presentation $S^{\prime} \rightarrow S$ and $x^{\prime} \in(G / N)_{\text {ant }}\left(S^{\prime}\right), y^{\prime} \in F\left(S^{\prime}\right)$ such that $g^{\prime}=x^{\prime} y^{\prime}$ in $(G / N)\left(S^{\prime}\right)$. Moreover, in view of Lemma 4.1, $x^{\prime}$ lifts to some $x^{\prime \prime} \in G_{\text {ant }}\left(S^{\prime \prime}\right)$, where $S^{\prime \prime} \rightarrow S^{\prime}$ is faithfully flat of finite presentation. So $g x^{\prime \prime-1} \in G\left(S^{\prime \prime}\right)$ lifts $y^{\prime}$, and hence $g \in G_{\text {ant }}\left(S^{\prime \prime}\right) H\left(S^{\prime \prime}\right)$.

If $G$ is smooth and $k$ is perfect, then $N$ may be chosen smooth by Remark 4.3; also, $F$ may be chosen smooth by Remark 3.3. Then $H$ is smooth as well.

We now derive from Theorem 4.7 a generalization of our main Theorem 1.1, under the additional assumption of characteristic 0 (then reductivity is equivalent to linear reductivity, also known as full reducibility):

Corollary 4.8. Every extension (1) with reductive quotient group $Q$ is quasisplit when $\operatorname{char}(k)=0$.

Proof. Choose an affine subgroup $H \subset G$ such that $G=G_{\text {ant }} \cdot H$ and denote by $R_{u}(H)$ its unipotent radical. By a result of Mostow [1956, Theorem 6.1], $H$ has a Levi subgroup, i.e., a fully reducible algebraic subgroup $L$ such that $H=R_{u}(H) \rtimes L$. Note that $R_{u}(H)$ is normal in $G$, since it is normalized by $H$ and centralized by $G_{\text {ant }}$. It follows that $G_{\text {ant }} \cdot R_{u}(H)$ is normal in $G$, and $G=\left(G_{\text {ant }} \cdot R_{u}(H)\right) \cdot L$. Also, note that the quotient map $f: G \rightarrow Q$ sends $G_{\text {ant }}$ to $e_{Q}$ (since $Q$ is affine), and $R_{u}(H)$ to $e_{Q}$ as well (since $Q$ is reductive). It follows that the sequence

$$
1 \longrightarrow N \cap L \longrightarrow L \stackrel{f}{\longrightarrow} Q \longrightarrow 1
$$

is exact, where $N=\operatorname{Ker}(f)$. If $N \cap L$ has a quasicomplement $H$ in $L$, then $H$ is a quasicomplement to $N$ in $G$ (as follows, e.g., from Lemma 2.1 (v)). Thus, we may assume that $G$ is reductive. Since every quasicomplement to $N^{0}$ in $G$ is a quasicomplement to $N$, we may further assume that $N$ is connected.

We have a canonical decomposition

$$
G^{0}=D\left(G^{0}\right) \cdot R\left(G^{0}\right),
$$

where the derived subgroup $D\left(G^{0}\right)$ is semisimple, the radical $R\left(G^{0}\right)$ is a central torus, and $D\left(G^{0}\right) \cap R\left(G^{0}\right)$ is finite (see, e.g., [SGA $3_{\text {III }}$ 1970, XXII.6.2.4]). Thus, $G=D\left(G^{0}\right) \cdot\left(R\left(G^{0}\right) \cdot F\right)$, where $F \subset G$ is a quasicomplement to $G^{0}$. Likewise, $N=D(N) \cdot R(N)$, where $D(N) \subset D\left(G^{0}\right), R(N) \subset R\left(G^{0}\right)$ and both are normal in $G$. Denote by $S$ the neutral component of the centralizer of $D(N)$ in $D\left(G^{0}\right)$. Then $S$ is a normal semisimple subgroup of $G$, and a quasicomplement to $D(N)$ 
in $D\left(G^{0}\right)$. If $R(N)$ admits a quasicomplement $T$ in $R\left(G^{0}\right) \cdot F$, then one readily checks that $S \cdot T$ is a quasicomplement to $N$ in $G$. As a consequence, we may replace $G$ with $R\left(G^{0}\right) \cdot F$, and hence assume that $G^{0}$ is a torus.

Denote by $X^{*}\left(G^{0}\right)$ the character group of $G_{\bar{k}}^{0}$; this is a free abelian group of finite rank, equipped with a continuous action of $F(\bar{k}) \rtimes \Gamma$, where $\Gamma$ denotes the absolute Galois group of $k$. Moreover, we have a surjective homomorphism $\rho: X^{*}\left(G^{0}\right) \rightarrow X^{*}(N)$, equivariant for $F(\bar{k}) \rtimes \Gamma$. Thus, $\rho$ splits over the rationals, and hence there exists a subgroup $\Lambda \subset X^{*}\left(G^{0}\right)$, stable by $F(\bar{k}) \rtimes \Gamma$, which is mapped isomorphically by $\rho$ to a subgroup of finite index of $X^{*}(N)$. The quotient $X^{*}\left(G^{0}\right) / \Lambda$ corresponds to a subtorus $H \subset G^{0}$, normalized by $G$, which is a quasicomplement to $N$ in $G^{0}$. So $H \cdot F$ is the desired quasicomplement to $N$ in $G$.

Remark 4.9. Corollary 4.8 does not extend to positive characteristics, due to the existence of groups without Levi subgroups (see [Conrad et al. 2015, Appendix A.6; McNinch 2010, Section 3.2]). As a specific example, when $k$ is perfect of characteristic $p>0$, there exists a nonsplit extension of algebraic groups

$$
1 \longrightarrow V \longrightarrow G \stackrel{f}{\longrightarrow} \mathrm{SL}_{2} \longrightarrow 1,
$$

where $V$ is a vector group on which $\mathrm{SL}_{2}$ acts linearly via the Frobenius twist of its adjoint representation. We show that this extension is not quasisplit. Otherwise, let $H$ be a quasicomplement to $N$ in $G$. Then so is the reduced neutral component of $H$, and hence we may assume that $H$ is smooth and connected. The quotient map $f$ restricts to an isogeny $H \rightarrow \mathrm{SL}_{2}$, and hence to an isomorphism. Thus, the above extension is split, a contradiction.

Next, we obtain an analogue of the Levi decomposition (see [Mostow 1956] again) for possibly nonlinear algebraic groups:

Corollary 4.10. Let $G$ be an algebraic group over a field of characteristic 0 . Then $G=R \cdot S$, where $R \subset G$ is the largest connected solvable normal subgroup, and $S \subset G$ is an algebraic subgroup such that $S^{0}$ is semisimple; also, $R \cap S$ is finite.

Proof. By a standard argument, $G$ has a largest connected solvable normal subgroup $R$. The quotient $G / R$ is affine, since $R \supset G_{\text {ant }}$. Moreover, $R / G_{\text {ant }}$ contains the radical of $G / G_{\text {ant }}$, and hence $(G / R)^{0}$ is semisimple. In particular, $G / R$ is reductive. So Corollary 4.8 yields the existence of the quasicomplement $S$.

Remark 4.11. One may ask for a version of Corollary 4.10 in which the normal subgroup $R$ is replaced with an analogue of the unipotent radical of a linear algebraic group, and the quasicomplement $S$ is assumed to be reductive. But such a version would make little sense when $G$ is an antiaffine semiabelian variety (for example, when $G$ is the extension of an abelian variety $A$ by $\mathbb{G}_{m}$, associated with an algebraically trivial line bundle of infinite order on $A$ ). Indeed, such a group $G$ 
has a largest connected reductive subgroup: its maximal torus, which admits no quasicomplement.

Also, recall that the radical $R$ may admit no complement in $G$, e.g., when $G=\mathrm{GL}_{n}$ with $n \geq 2$.

Finally, one may also ask for the uniqueness of a minimal quasicomplement in Corollary 4.10, up to conjugacy in $R(k)$ (as for Levi complements, see [Mostow 1956, Theorem 6.2]). But this fails when $k$ is algebraically closed and $G$ is the semidirect product of an abelian variety $A$ with a group $F$ of order 2. Denote by $\sigma$ the involution of $A$ induced by the nontrivial element of $F$; then $R=A$, and the complements to $R$ in $G$ are exactly the subgroups generated by the involutions $x \sigma$ where $x \in A^{-\sigma}(k)$, i.e., $\sigma(x)=x^{-1}$. The action of $R(k)$ on complements is given by $y x \sigma y^{-1}=x y \sigma(y)^{-1} \sigma$; moreover, the homomorphism $A \rightarrow A^{-\sigma}, y \mapsto y \sigma(y)^{-1}$ is generally not surjective. This holds for example when $A=(B \times B) / C$, where $B$ is a nontrivial abelian variety, $C$ is the subgroup of $B \times B$ generated by $\left(x_{0}, x_{0}\right)$ for some $x_{0} \in B(k)$ of order 2 , and $\sigma$ arises from the involution $(x, y) \mapsto\left(y^{-1}, x^{-1}\right)$ of $B \times B$; then $A^{-\sigma}$ has 2 connected components.

Another consequence of Theorem 4.7 concerns the case where $k$ is finite; then every antiaffine algebraic group is an abelian variety (see [Brion 2009, Proposition 2.2]). This yields readily:

Corollary 4.12. Let $G$ be an algebraic group over a finite field. Then $G$ sits in an extension of algebraic groups

$$
1 \longrightarrow F \longrightarrow A \times H \longrightarrow G \longrightarrow 1,
$$

where $F$ is finite, $A$ is an abelian variety, and $H$ is affine. If $G$ is smooth, then $H$ may be chosen smooth as well.

Returning to an arbitrary base field, we finally obtain the existence of equivariant compactifications of homogeneous spaces:

Theorem 4.13. Let $G$ be an algebraic group, and $H$ a closed subgroup. Then there exists a projective scheme $X$ equipped with an action of $G$, and an open $G$-equivariant immersion $G / H \hookrightarrow X$ with schematically dense image.

Proof. When $G$ is affine, this follows from a theorem of Chevalley asserting that $H$ is the stabilizer of a line $L$ in a finite-dimensional $G$-module $V$ (see [SGA $3_{\text {I }}$ 1970, VIB.11.16]). Indeed, one may take for $X$ the closure of the $G$-orbit of $L$ in the projective space of lines of $V$; then $X$ satisfies the required properties in view of [Demazure and Gabriel 1970, III.3.5.2]. Note that $X$ is equipped with an ample $G$-linearized invertible sheaf.

When $G$ is proper, the homogeneous space $G / H$ is proper as well, and hence is projective by [Raynaud 1970, Corollary VI.2.6] (alternatively, this follows from the structure of $X$ described in Remark 4.5). 
In the general case, Theorem 4.2 yields an affine normal subgroup $N$ of $G$ such that $G / N$ is proper. Then $N \cdot H$ is a subgroup of $G$, and $G /(N \cdot H)$ is proper as well, hence projective. It suffices to show the existence of a projective scheme $Y$ equipped with an action of $N \cdot H$, an open immersion $(N \cdot H) / H \rightarrow Y$ with schematically dense image, and a $N \cdot H$-linearized ample line bundle: indeed, by [Mumford et al. 1994, Proposition 7.1] applied to the projection $G \times Y \rightarrow Y$ and the $N \cdot H$-torsor $G \rightarrow G /(N \cdot H)$, the associated fiber bundle $G \times{ }^{N \cdot H} Y$ yields the desired equivariant compactification. In view of Chevalley's theorem used in the first step, it suffices in turn to check that $N \cdot H$ acts on $(N \cdot H) / H$ via an affine quotient group; equivalently, $(N \cdot H)_{\text {ant }} \subset H$.

By Lemma $4.1,(N \cdot H)_{\text {ant }}$ is a quotient of $(N \rtimes H)_{\text {ant }}$. The latter is the fiber at the neutral element of the affinization morphism $N \rtimes H \rightarrow \operatorname{Spec} \mathcal{O}(N \rtimes H)$. Also, $N \rtimes H \cong N \times H$ as schemes, $N$ is affine and the affinization morphism commutes with products; thus, $(N \rtimes H)_{\text {ant }}=H_{\text {ant }}$. As a consequence, $(N \cdot H)_{\text {ant }}=H_{\text {ant }}$; this completes the proof.

Remark 4.14. If $\operatorname{char}(k)=0$, the equivariant compactification $X$ of Theorem 4.13 may be taken smooth, as follows from the existence of an equivariant desingularization (see [Kollár 2007, Proposition 3.9.1, Theorem 3.36]).

In arbitrary characteristics, $X$ may be taken normal if $G$ is smooth. Indeed, the $G$-action on any equivariant compactification $X$ stabilizes the reduced subscheme $X_{\text {red }}$ (since $G \times X_{\text {red }}$ is reduced), and lifts to an action on its normalization $\widetilde{X}$ (since $G \times \tilde{X}$ is normal). But the existence of regular compactifications (equivariant or not) is an open question.

Over any imperfect field $k$, there exist smooth connected algebraic groups $G$ having no smooth compactification. Indeed, we may take for $G$ the subgroup of $\mathbb{G}_{a} \times$ $\mathbb{G}_{a}$ defined by $y^{p}-y-t x^{p}=0$, where $p:=\operatorname{char}(k)$ and $t \in k \backslash k^{p}$. This is a smooth affine curve, and hence has a unique regular compactification $X$. One checks that $X$ is the curve $\left(y^{p}-y z^{p-1}-t x^{p}=0\right) \subset \mathbb{P}^{2}$, which is not smooth at its point at infinity.

\section{Acknowledgements}

I warmly thank Giancarlo Lucchini Arteche for very helpful e-mail discussions, and Zinovy Reichstein for his interest and for pointing out several references. Many thanks also to the referee for a careful reading of this note, and useful comments.

\section{References}

[Arima 1960] S. Arima, "Commutative group varieties", J. Math. Soc. Japan 12 (1960), 227-237. MR 25 \#79 Zbl 0113.36307

[Borel and Serre 1964] A. Borel and J.-P. Serre, "Théorèmes de finitude en cohomologie galoisienne", Comment. Math. Helv. 39 (1964), 111-164. MR 31 \#5870 Zbl 0143.05901 
[Bosch et al. 1990] S. Bosch, W. Lütkebohmert, and M. Raynaud, Néron models, Ergebnisse der Mathematik und ihrer Grenzgebiete (3) 21, Springer, Berlin, 1990. MR 91i:14034 Zbl 0705.14001

[Brion 2009] M. Brion, "Anti-affine algebraic groups", J. Algebra 321:3 (2009), 934-952. MR 2009j: 14059 Zbl 1166.14029

[Chernousov et al. 2008] V. Chernousov, P. Gille, and Z. Reichstein, "Reduction of structure for torsors over semilocal rings", Manuscripta Math. 126:4 (2008), 465-480. MR 2009f:14090 Zbl 1157.14028

[Conrad et al. 2015] B. Conrad, O. Gabber, and G. Prasad, Pseudo-reductive groups, 2nd ed., New Mathematical Monographs 17, Cambridge University Press, 2015. MR 2011k:20093 Zbl 1314.20037

[Demazure and Gabriel 1970] M. Demazure and P. Gabriel, Groupes algébriques, I: Géométrie algébrique, généralités, groupes commutatifs, Masson, Paris, 1970. MR 46\#1800 Zbl 0203.23401

[Gabber 2012] O. Gabber, "On pseudo-reductive groups and compactification theorems", Oberwolfach Reports 9 (2012), 2371-2374. MR 3156730

[Gabber et al. 2014] O. Gabber, P. Gille, and L. Moret-Bailly, "Fibrés principaux sur les corps valués Henséliens", Algebr. Geom. 1:5 (2014), 573-612. MR 3296806

[Kollár 2007] J. Kollár, Lectures on resolution of singularities, Annals of Mathematics Studies 166, Princeton University Press, 2007. MR 2008f:14026 Zbl 1113.14013

[Lang 1956] S. Lang, "Algebraic groups over finite fields", Amer. J. Math. 78 (1956), 555-563. MR 19,174a Zbl 0073.37901

[Lötscher et al. 2013] R. Lötscher, M. MacDonald, A. Meyer, and Z. Reichstein, "Essential pdimension of algebraic groups whose connected component is a torus", Algebra Number Theory 7:8 (2013), 1817-1840. MR 3134035 Zbl 1288.20061

[Lucchini Arteche 2015a] G. Lucchini Arteche, "Extensions of algebraic groups with finite quotient and non-abelian 2-cohomology”, preprint, 2015. arXiv 1503.06582

[Lucchini Arteche 2015b] G. Lucchini Arteche, "Groupe de Brauer non ramifié algébrique des espaces homogènes", Transform. Groups 20:2 (2015), 463-493. MR 3348564 Zbl 06458683

[Matsumura and Oort 1967] H. Matsumura and F. Oort, "Representability of group functors, and automorphisms of algebraic schemes", Invent. Math. 4 (1967), 1-25. MR 36 \#181 Zbl 0173.22504

[McNinch 2010] G. J. McNinch, "Levi decompositions of a linear algebraic group", Transform. Groups 15:4 (2010), 937-964. MR 2012b:20110 Zbl 1221.20034

[Milne 2013] J. S. Milne, "A proof of the Barsotti-Chevalley theorem on algebraic groups", preprint, 2013. arXiv 1311.6060v2

[Mostow 1956] G. D. Mostow, "Fully reducible subgroups of algebraic groups", Amer. J. Math. 78 (1956), 200-221. MR 19,1181f Zbl 0073.01603

[Mumford et al. 1994] D. Mumford, J. Fogarty, and F. Kirwan, Geometric invariant theory, 3rd ed., Ergebnisse der Mathematik und ihrer Grenzgebiete (2) 34, Springer, Berlin, 1994. MR 95m:14012 Zbl 0797.14004

[Platonov 1966] V. P. Platonov, “Теория алгебраических линейных групп и периодические группы”, Izv. Akad. Nauk SSSR Ser. Mat. 30:3 (1966), 573-620. Translated as "Theory of algebraic linear groups and periodic groups", pp. 61-110 in Seven papers on algebra, Transl. Amer. Math. Soc. (2) 69, Amer. Math. Soc., Providence, RI, 1968. MR 33 \#7428 Zbl 0146.04405

[Raynaud 1970] M. Raynaud, Faisceaux amples sur les schémas en groupes et les espaces homogènes, Lecture Notes in Math. 119, Springer, Berlin, 1970. MR 41 \#5381 Zbl 0195.22701

[Rosenlicht 1956] M. Rosenlicht, "Some basic theorems on algebraic groups", Amer. J. Math. 78 (1956), 401-443. MR 18,514a Zbl 0073.37601 
[Rosenlicht 1961] M. Rosenlicht, "Toroidal algebraic groups", Proc. Amer. Math. Soc. 12 (1961), 984-988. MR 24 \#A3162 Zbl 0107.14703

[Sancho and Sancho 2009] C. Sancho de Salas and F. Sancho de Salas, "Principal bundles, quasiabelian varieties and structure of algebraic groups", J. Algebra 322:8 (2009), 2751-2772. MR 2010m: 14058 Zbl 1191.14055

[SGA $3_{\text {I }}$ 1970] M. Demazure and A. Grothendieck, Schémas en groupes, Tome I: Propriétés générales des schémas en groupes, Exposés I-VII (Séminaire de Géométrie Algébrique du Bois Marie 19621964), Lecture Notes in Math. 151, Springer, Berlin, 1970. MR 43 \#223a Zbl 0207.51401

[SGA 3 II 1970] M. Demazure and A. Grothendieck, Schémas en groupes, Tome II: Groupes de type multiplicatif, et structure des schémas en groupes généraux, Exposés VIII-XVIII (Séminaire de Géométrie Algébrique du Bois Marie 1962-1964), Lecture Notes in Math. 152, Springer, Berlin, 1970. MR 43 \#223b Zbl 0209.24201

[SGA 3 III 1970] M. Demazure and A. Grothendieck, Schémas en groupes, Tome III: Structure des schémas en groupes réductifs, Exposés XIX-XXVI (Séminaire de Géométrie Algébrique du Bois Marie 1962-1964), Lecture Notes in Math. 153, Springer, Berlin, 1970. MR 43 \#223c Zbl 0212.52810

[Totaro 2013] B. Totaro, "Pseudo-abelian varieties", Ann. Sci. Éc. Norm. Supér. (4) 46:5 (2013), 693-721. MR 3185350 Zbl 1286.14061

Received May 27, 2015. Revised July 12, 2015.

MICHEL BRION

INSTITUT FOURIER

UNIVERSITÉ DE GRENOBLE I (JOSEPH FOURIER)

100 RUE DES MATHÉMATIQUES

38402 SAINT-MARTIN D'HÈrES CEDEX

FRANCE

michel.brion@ujf-grenoble.fr 


\title{
PACIFIC JOURNAL OF MATHEMATICS
}

\author{
msp.org/pjm
}

Founded in 1951 by E. F. Beckenbach (1906-1982) and F. Wolf (1904-1989)

\section{EDITORS}

Don Blasius (Managing Editor)

Department of Mathematics

University of California

Los Angeles, CA 90095-1555

blasius@math.ucla.edu

\author{
Paul Balmer \\ Department of Mathematics \\ University of California \\ Los Angeles, CA 90095-1555 \\ balmer@math.ucla.edu \\ Robert Finn \\ Department of Mathematics \\ Stanford University \\ Stanford, CA 94305-2125 \\ finn@math.stanford.edu \\ Sorin Popa \\ Department of Mathematics \\ University of California \\ Los Angeles, CA 90095-1555 \\ popa@math.ucla.edu
}

\author{
Vyjayanthi Chari \\ Department of Mathematics \\ University of California \\ Riverside, CA 92521-0135 \\ chari@math.ucr.edu \\ Kefeng Liu \\ Department of Mathematics \\ University of California \\ Los Angeles, CA 90095-1555 \\ liu@math.ucla.edu \\ Jie Qing \\ Department of Mathematics \\ University of California \\ Santa Cruz, CA 95064 \\ qing@ cats.ucsc.edu
}

\section{PRODUCTION}

Silvio Levy, Scientific Editor, production@msp.org

\section{SUPPORTING INSTITUTIONS}

ACADEMIA SINICA, TAIPEI

CALIFORNIA INST. OF TECHNOLOGY

INST. DE MATEMÁTICA PURA E APLICADA

KEIO UNIVERSITY

MATH. SCIENCES RESEARCH INSTITUTE

NEW MEXICO STATE UNIV.

OREGON STATE UNIV.

\author{
STANFORD UNIVERSITY \\ UNIV. OF BRITISH COLUMBIA \\ UNIV. OF CALIFORNIA, BERKELEY \\ UNIV. OF CALIFORNIA, DAVIS \\ UNIV. OF CALIFORNIA, LOS ANGELES \\ UNIV. OF CALIFORNIA, RIVERSIDE \\ UNIV. OF CALIFORNIA, SAN DIEGO \\ UNIV. OF CALIF., SANTA BARBARA
}

\author{
Daryl Cooper \\ Department of Mathematics \\ University of California \\ Santa Barbara, CA 93106-3080 \\ cooper@math.ucsb.edu \\ Jiang-Hua Lu \\ Department of Mathematics \\ The University of Hong Kong \\ Pokfulam Rd., Hong Kong \\ jhlu@maths.hku.hk \\ Paul Yang \\ Department of Mathematics \\ Princeton University \\ Princeton NJ 08544-1000 \\ yang@math.princeton.edu
}

These supporting institutions contribute to the cost of publication of this Journal, but they are not owners or publishers and have no responsibility for its contents or policies.

See inside back cover or msp.org/pjm for submission instructions.

The subscription price for 2015 is US \$420/year for the electronic version, and \$570/year for print and electronic.

Subscriptions, requests for back issues and changes of subscribers address should be sent to Pacific Journal of Mathematics, P.O. Box 4163, Berkeley, CA 94704-0163, U.S.A. The Pacific Journal of Mathematics is indexed by Mathematical Reviews, Zentralblatt MATH, PASCAL CNRS Index, Referativnyi Zhurnal, Current Mathematical Publications and Web of Knowledge (Science Citation Index).

The Pacific Journal of Mathematics (ISSN 0030-8730) at the University of California, c/o Department of Mathematics, 798 Evans Hall \#3840, Berkeley, CA 94720-3840, is published twelve times a year. Periodical rate postage paid at Berkeley, CA 94704, and additional mailing offices. POSTMASTER: send address changes to Pacific Journal of Mathematics, P.O. Box 4163, Berkeley, CA 94704-0163.

PJM peer review and production are managed by EditFLOW ${ }^{\circledR}$ from Mathematical Sciences Publishers.

\section{PUBLISHED BY}

\section{mathematical sciences publishers \\ nonprofit scientific publishing}

http://msp.org/

(C) 2015 Mathematical Sciences Publishers 


\title{
PACIFIC JOURNAL OF MATHEMATICS
}

\author{
Volume 279 No. 1-2 December 2015
}

In memoriam: Robert Steinberg

Robert Steinberg (1922-2014): In memoriam V. S. VARADARAJAN

Cellularity of certain quantum endomorphism algebras

HENNING H. ANDERSEN, GUSTAV I. LEHRER and RUIBIN ZHANG

Lower bounds for essential dimensions in characteristic 2 via orthogonal representations ANTONIO BABIC and VLADIMIR CHERNOUSOV

Cocharacter-closure and spherical buildings

Michael Bate, Sebastian Herpel, Benjamin Martin and Gerhard RöHrLe

Embedding functor for classical groups and Brauer-Manin obstruction

Eva Bayer-Fluckiger, Ting-Yu LeE and Raman Parimala

On maximal tori of algebraic groups of type $G_{2}$

Constantin Beli, PhilipPe Gille and Ting-Yu LeE

On extensions of algebraic groups with finite quotient

MICHEL BRION

Essential dimension and error-correcting codes

SHANE CERNELE and ZiNOVy REICHSTEIN

Notes on the structure constants of Hecke algebras of induced representations of finite Chevalley groups

Charles W. CuRTis

Complements on disconnected reductive groups

FRANÇOIS DIGNE and JEAN MICHEL

Extending Hecke endomorphism algebras

Jie Du, Brian J. Parshall and LeOnard L. SCOTT

Products of partial normal subgroups

ELLEN HENKE

Lusztig induction and $\ell$-blocks of finite reductive groups

RADHA KESSAR and GUNTER MALLE

Free resolutions of some Schubert singularities

Manoj Kummini, Venkatramani Lakshmibai, Pramathanath Sastry and C. S. Seshadri

Free resolutions of some Schubert singularities in the Lagrangian Grassmannian

VenKatramani LAKSHMibai and ReUVEN HODGES

Distinguished unipotent elements and multiplicity-free subgroups of simple algebraic groups

Martin W. Liebeck, Gary M. Seitz and Donna M. Testerman

Action of longest element on a Hecke algebra cell module

GEORGE LUSZTIG

Generic stabilisers for actions of reductive groups

BENJAMIN MARTIN

On the equations defining affine algebraic groups

VLADIMIR L. POPOV

Smooth representations and Hecke modules in characteristic $p$

PETER SCHNEIDER

On CRDAHA and finite general linear and unitary groups

BHAMA SRINIVASAN

Weil representations of finite general linear groups and finite special linear groups PHAM HUU TIEP

The pro- $p$ Iwahori Hecke algebra of a reductive $p$-adic group, $\mathrm{V}$ (parabolic induction) MARIE-FRANCE VIGNÉRAS

Acknowledgement 